\title{
LA INVESTIGACIÓN CUALITATIVA EN EDUCACIÓN Y LA RELACIÓN CON EL CAMPO PEDAGÓGICO
}

Por:

\author{
Alberto Sarmiento Castro'
}

\section{RESUMEN}

La investigación en el campo educativo se define como un largo camino que intenta comprender el papel de los sujetos, la interacción entre las personas, experimentando la incertidumbre, la duda y la certeza, pero a través de sus hallazgos construye un mundo de conocimiento. La investigación cualitativa está asociada a explorar dentro de un mundo explicativo (positivista) o interpretativo o comprensivo (cualitativo social) y vivir construyendo los procedimientos de la investigación desde lo pedagógico, el conocimiento mismo de los procedimientos investigativos mediados por la etnografía, la hermenéutica, la fenomenología y la teoría fundada.

Palabras claves: investigación cualitativa, campo pedagógico, investigación en educación.

"Freud indicaba en su autografía que le movía una especie de curiosidad dirigida:

"más hacia asuntos humanos que hacia objetos naturales".

Lo que más llama la atención de sus cartas

es su fascinación ante el mundo

llena de debilidades de los seres humanos

y la comprensión".

Howard Gardner

Mentes creadoras

Selene. Segunda expedición de pleyade.

Fundación FES. MEN 1.998

\section{INTRODUCCIÓN}

La investigación puede ser interpretada desde nuestras miradas y según el contexto donde se utiliza. Los animales son investigadores naturales del entorno donde se mueven y de ese ir o venir, van construyendo una huella/camino-rastreo y dependen de ese aprendizaje para sobrevivir. La investigación está asociada a la capacidad de explorar, a curiosear y de pistas, a través de los elementos corporales y el uso de los sentidos. Los seres humanos desde la niñez están en capacidad de curiosear, de explorar el ambiente, las palabras, de jugar con las preguntas y los signos (codificación-decodificación), de desarmar objetos y archivar recuerdos que van sembrando la duda y la certeza para aprender a desenvolverse en su mundo y poder interactuar con su propio medio.

A medida que avanza en el largo caminar de la vida, dispone de la capacidad lógica para comprender el mundo que intenta identificar para su proyecto (introspección), construye teorías sobre el acontecer y aprovecha al máximo de la información que dispone, entra al campo del conocimiento, asume, rechaza o se arriesga para abrir camino en situaciones complejas de las relaciones de los imaginarios del hombre por posibilitar un mundo más real; derivada de las relaciones sujeto-objeto que permiten focalizarlo en un mundo subjetivo-intersubjetivo u objetivo, concreto, demostrativo, capaz de generar hechos nuevos.

La problemática desde lo objetivo o lo subjetivo, es uno de los temas que más contradicciones ha generado en el campo de la filosofía, de la ciencia, el arte o la psicología y todas las otras disciplinas que involucren

\footnotetext{
'Profesor área de Filosofía. Departamento de Humanidades. Universidad Francisco de Paula Santander E-mail: alsaca57@hotmail.com
} 
La investigación cualitativa en educación y la relación con el campo pedagógico

Alberto Sarmiento Castro

los problemas de los saberes construidos por el hombre. Lo objetivo es lo material y de lo real, posiblemente tiene caminos demostrativos; se asume lo objetivo, en una actitud fuera del sentido personal (papel neutro), alejado desde la mirada del objeto y el uso propio de la razón (acto de raciocinio).

Lo subjetivo está relacionado con la mirada propia del sujeto, con lo que desea y la interacción entre las personas, guiadas por el impulso afectivo (percepción, interpretación del hecho) y no por la razón, alejado del hecho intersubjetivo de construir los niveles comprensivos de los hechos propios de los seres humanos con teorías o conceptos, de experimentación, de fracasos, de incertidumbres y sobre todo, de pasión, de reflexiones (duda, certeza) y acciones sobre el mundo en el que participa y toma decisiones con otros.

Aquí, en este momento, cabe preguntar: ¿̇Cuál es el quehacer investigativo en la educación y su relación con la pedagogía?

Todos los seres humanos están en posibilidades de conocer los hechos o las cosas que se relacionan con el mundo, porque se tiene una sabiduría (cotidianidad, aprendizaje), una cultura y la facultad de conocer y profundizar con lo conocido (acto cognitivo), este hecho se desarrolla en un proceso constante que, a lo largo de la vida y/o campo científico, se va reconociendo el objeto de la investigación para alcanzar una mayor claridad -confiabilidad- entre el quehacer investigativo en la educación y su relación con la pedagogía. Esa relación teórica o metodológica se deriva de:

- Desde los paradigmas de la investigación cuantitativa (ciencias positivistas: explicativo) o cualitativa (ciencias sociales: interpretativo o comprensivo).

- Desde la relación de la investigación de las ciencias sociales y el campo pedagógico, y

- Cuál es el modo de construir el conocimiento el investigador.
1. Desde los paradigmas de la investigación cuantitativa (ciencias positivistas: explicativo) o cualitativo (ciencias sociales: interpretativo o comprensivo) Briones 1.995, Cerda 1.995.

\section{PARADIGMA CUANTITATIVO (EXPLICATIVO)}

- Parte de la existencia de cosas reales e independientes de la conciencia. Es fáctico y analítico.

- Tiene como base los referentes empíricos para afirmar el resultado de las investigaciones.

- Mantiene los postulados de la ciencia (explicativo) como el método científico, determinante, claro y explicativo.

- La explicación es predictiva y parte siempre de factores causa-efecto que pueden basarse en la teoría. Presupone los resultados cuando constituyen una ley o hecho.

- Le interesa medir la probabilidad que ocurrirá en un fenómeno determinado (datos, hechos, estadística).

- La explicación supone la tarea previa de describir el fenómeno a explicar (deducción) y acude a las observaciones experimentales.

- La relación investigador-objeto de estudio se da en una realidad externa mediada por la formación del método científico de las ciencias positivas.

- Los hechos a investigar siempre están determinados por la elección del diseño de la investigación y la evaluación de los resultados obtenidos por el investigador.

- Todo hecho empírico se relaciona con las teorías y las hipótesis que permiten proporcionar conocimientos más amplios y profundos. 
La investigación cualitativa en educación y la relación con el campo pedagógico

Alberto Sarmiento Castro

- Tiene como base la objetividad científica, por lo que el hecho es un dato real, claro y objetivo y utiliza métodos extensivos y probabilísticos.

\section{PARADIGMA CUALITATIVO (INTERPRETATIVO O COMPRENSIVO)}

- Interpreta y comprende la conducta de las personas dentro de su propia cultura de la comunidad, del grupo o individual. Hace una lectura de la realidad holística.

- Quien investiga no es sólo observador, sino instrumento de transformación que identifica con el grupo el problema y ayuda a solucionarlo y acude a observaciones naturales.

- A partir de la interacción define y redefine las situaciones en las cuales viven o actúan las personas para resolver sus problemas.

- Permite a los actores establecer una relación más directa de cómo viven sus miembros, utiliza modelos intensivos, profundos.

- No existe una sola verdad, sino que surge como la configuración de las diversas formas de ver la persona su propio mundo y las relaciones con otro.

- Es una interrelación que implica entre sujeto y objeto desde las acciones mismas de la investigación.

- Los hechos no parten de los resultados obtenidos, sino de las características propias del grupo, por ello no busca explicación de los hechos, sino interpretarlos.

- El conocimiento parte sobre el comportamiento humano y la forma como funcionan los grupos y las comunidades en un contexto de condiciones culturales.
- Establece una relación intersubjetiva desde el diálogo de los sujetos que se investigan; es constructivista y dialógica.

\section{DESDE LA RELACIÓN DE LA INVESTIGACIÓN DE LAS CIENCIAS SOCIALES Y EL CAMPO PEDAGÓGICO}

La relación de la investigación de las Ciencias Sociales y el campo pedagógico, parten de la estructura conceptual y óptica del investigador, por ello configura su mirada dentro del entorno social y desde allí su objeto del conocimiento; por otro lado, en lo pedagógico está centrado en las formas de aprender y sus prácticas pedagógicas, concebidas de las relaciones complejas, multidisciplinarias, transversales e intersubjetivas constituidas principalmente por la interacción cotidiana de los sujetos que interactúan en el medio social o educativo de la colectividad humana.

Esta mirada pretende reconstruir, construir, interpretar y comprender el fenómeno educativo.

Otro hecho de definición de la pedagogía en el acto de la investigación, es posible, si hay un sujeto que investiga, que pregunta sobre el objeto investigado y se establecen unas teorías y metodologías trazadas por las ciencias y uso racional del sujeto investigador (neutralidad del objeto - sujeto) y no exige contacto directo con la población estudiada para facilitar la neutralidad y la objetividad.

Ahora el investigador desde la pedagogía, encierra una relación diferente a sujeto y objeto de estudio; el marco teórico está construido desde el interior del sujeto y la relación con otros sujetos (intersubjetividad), el sujeto-cognoscente se convierte a su vez en un elemento compartido, por ser el hombre un ser biológico, un ser cognoscente, un ser que se desenvuelve en un saber hacer y ama lo que hace, es 
La investigación cualitativa en educación y la relación con el campo pedagógico

Alberto Sarmiento Castro

poseedor de un lenguaje y una cultura. Es así, que en el acto educativo y social el sujeto-objeto se dimensionan en un mismo acto a investigar y participan conjuntamente investigador-comunidad para favorecer los aspectos de interpretación de las relaciones interpersonales o grupales e involucra las prácticas educativas en un campo de aprendizaje y acciones pedagógicas del maestro-estudiante en el encuentro de la escuela, en un mundo complejo y multidimensional que permite conocer la realidad social y buscan caminos a su propia problemática.

Ahora el problema, se aborda desde el hombre mismo, sus relaciones, grados de organización y su funcionalidad en un determinado contexto comunitario, donde fluyen los lenguajes, sus símbolos - reglas para alcanzar las metas y los objetivos propuestos o en contravía del sistema educativo. Es así, que la investigación educativa, fija su mirada en los procesos pedagógicos (acto de enseñar, aprender, niveles de conceptualización teórica y su nivel metodológico para alcanzar la enseñabilidad de las disciplinas) de los problemas a diario que enfrentan los diferentes agentes educativos, en el fortalecimiento por el trabajo, agregados a los valores humanos, a la construcción de saberes y la manera de actuar para resolver los conflictos propios del campo social y educativo.

Es enfocar el campo de la investigación cualitativa en comprender mejor el hecho pedagógico (instrucciónenseñanza - aprendizaje), para lograr que los individuos piensen por sí mismos y develar la construcción o desconstrucción de los saberes producidos por la humanidad. Es un acto que va abriendo caminos, que pregunta, duda, dialoga y comprende las relaciones del conocimiento (campo referencial) y es el maestro sujeto-objeto al mismo tiempo, permitiendo determinar el fenómeno problematizador dentro de las ciencias de la educación.

\section{¿CUÁL ES EL MODO DE CONSTRUIR EL CONOCIMIENTO EL INVESTIGADOR?}

Hasta aquí se ha hecho un recorrido de la investigación en las Ciencias Sociales, estableciendo una relación estrecha con los postulados positivistas y a su vez el papel de la investigación pedagógica desde los presupuestos de la subjetividad y permitir a si una visión más clara del método interpretativo y comprensivo de las ciencias de la educación.

Esta dialéctica argumentativa entre la continuidad y la discontinuidad, no es sino un dualismo de los acontecimientos que abordan un investigador. Por un lado la observación neutral, objetiva y completa de un hecho, está valorada por el enorme cúmulo de teorías estructuralistas que observan al sujeto, fuera del objeto. Por otro lado, la realidad está mediada por el sujeto, asume la riqueza de la incertidumbre, del conflicto, del caos y la complejidad, establecida en un mundo social o educativo; pero el investigador toma criterios de validación de uno u otro método, de acuerdo con la naturaleza que desea estudiar.

Estas notas precedentes discuten la especificidad o no de lo humano como objeto de conocimiento y en vez de separar los enfoques debe alimentar un hecho mixto, pero principalmente nos ubicamos en el campo cualitativo por aspectos intersubjetivos como es la educación y dentro de ella el acto pedagógico. Estos presupuestos se enmarcan:

- La realidad que pretende conocer está mediada por el sujeto que la conoce. Los objetos dependen de las características individuales, culturales o sociales de quien o quienes examinan las características examinadas.

- Las ciencias del espíritu conciben la realidad desde el sujeto mismo, no está en el exterior; por ello, su riqueza en la duda, el conflicto, la incertidumbre, el caos y la complejidad. 
La investigación cualitativa en educación y la relación con el campo pedagógico

Alberto Sarmiento Castro

- Se incluyen la dimensión humana de la subjetividad inherente a la naturaleza del hombre y la interacción con su mundo que le da sentido y significado a los actos humanos. Estos hechos posibilitan la crítica y la autorreflexión del investigador en la construcción del conocimiento.

- Los problemas de la investigación nacen de la realidad social y humana de los hombres y es aquí donde el investigador toma un lugar en el desarrollo del hecho a investigar para interpretar y comprender la naturaleza cultural de las personas, grupos o comunidad: es actor (investigador) y constructor de la realidad que conoce y transforma.

- Se recupera la subjetividad como espacio de construcción de la vida humana, y la reivindicación de la vida cotidiana como escenario para comprender la realidad sociocultural del hombre y esa opción de íntersubjetividad y de consenso van vínculos para acceder al conocimiento válido de la realidad humana (obsérvese el epígrafe de Freud. Gardner. Mentes creadoras. Francisco Cajiao. Selene. Segunda Expedición Pleyade 1.998).

- Estos enfoques teóricos de la investigación social cualitativa, están mediados por:

- Etnografía: una visión de lo humano desde la cultura. La cultura es un núcleo de análisis y se ocupa de la descripción de los estilos de vida de personas dentro de un ámbito del convivir. Nace en la Antropología y pone sus ojos en los logros humanos que tienen por marco la vida social (Taylor. 1994). Entiéndase la cultura como un sistema de cogniciones compartidas; el hombre (razón, intelecto, raciocinio) genera cultura a partir de un número finito de reglas. Lo importante de este hecho es captar el punto de vista, el sentido, las motivaciones, las intenciones y expectativas que las personas otorgan a sus propias acciones y el entorno cultural donde se produce o tiene lugar.

Etimológicamente etnografía, significa descripción del estilo de vida de un grupo humano que comparte un conjunto de características y a partir de ellas, establece un nuevo nivel de reflexión y análisis y mediante contrastes elabora sus propias teorías.

El ethnos, se caracteriza por un grupo humano que constituye una entidad, cuyas relaciones están reguladas por las costumbres o reciprocidad que hacen compartir algo de su estilo de vida (Geertz.1973) o como lo afirma Claude Levi-Strauss (1.983:2.000): "...el etnólogo intenta recuperar el significado, reconstruir el significado, por medios mecánicos, lo construye, lo desenvuelve y después de todo, él es un hombre y lo saborea".

- La hermenéutica: le interesan las percepciones, las sensaciones y las interpretaciones que hace el sujeto a partir del mundo exterior y le da sentido y tiene la capacidad de explicar o comprender los hechos que rodean al mundo y sus relaciones. Es un mundo que está ahí y en que vivimos y busca incrementar el entendimiento de otras culturas o grupos en el presente o el pasado.

Para Dilthey (1.833-1911) la hermenéutica es una herramienta o método de las ciencias del espíritu. Gadamer (1983) vuelve a una doctrina filosófica de la comprensión o investigación del ser histórico; busca entender con otros un texto y mediado por una visión horizontal del mismo (círculo hermenéutico) y a través del tiempo y el sujeto van a ser interpretados y revelados los hechos que le acontecen y restaura el sentido de la comprensión (sospecha y confianza).

La hermenéutica toma como modelo la historia y la autobiografía. Indaga aquello que es un hecho humano más allá de la contraposición, dándole sentido 
La investigación cualitativa en educación y la relación con el campo pedagógico

Alberto Sarmiento Castro

en el presente para interpretar, comprender y explicar los hechos que sucederán.

- La fenomenología e interaccionismo simbólico: trata de describir la experiencia sin acudir a explicaciones causales (Holstein y Gubriur. 1967), igualmente se focaliza sobre la experiencia vivida, por ello Martín Heidergger la llamó "La estructura de la vida" y es por ello que va desarrollando un marco de comprensión y de análisis de la realidad humana y esos poderes activos del conocimiento humano van construyendo los criterios de la conciencia, teoría desarrollada por Husserl, pero Schutz establece las bases fenomenológicas de las Ciencias Sociales: la intersubjetividad y la intersubjetividad en el mundo social.

Igualmente, unido a las consideraciones de la fenomenología, existe como base existencial de su teoría, cuatro elementos básicos (Van Mannen. 1990):

- El espacio vivido (espacialidad).

- El cuerpo vivido (corporeidad).

- El tiempo vivido (temporalidad) y

- Las relaciones humanas vividas (relacionalidad o comunalidad).

Estos elementos básicos giran alrededor de la búsqueda de ese acceso, a través de los siguientes pasos (Boya. 1990):

a. Intuición (desarrollo de los niveles de conciencia a través de ver y escuchar).

b. Análisis (involucra la identificación de la estructura del fenómeno bajo el estudio de la dialéctica conversación - diálogo).

c. Descripción (quien escucha, explora su propia experiencia del fenómeno).

d. Observación (aparición del fenómeno). e. Exploración en la conciencia (se reflexiona sobre las relaciones del fenómeno. Afinidades estructuales).

f. Suspensión de las creencias (juicio del fenómeno).

g. Interpretación de los significados ocultos o encubiertos (descubrir experiencias vividas y ser valoradas para informar la práctica y la ciencia).

Por otro lado, a comienzos del siglo XX, en Europa la fenomenología se orienta a las observaciones y el análisis hacia la forma como la gente experimenta y entiende subjetivamente el mundo y la construcción de su propia realidad social; este enfoque privilegia el lenguaje y la comunicación como medio por excelencia para la expresión de sentimientos y pensamientos.

También se le denomina interaccionismo simbólico (George Herbert Mead) el define que mediante la interacción social conocemos nuestro lugar en el mundo, la identidad de una persona para la construcción de ese mundo social (aprendizaje social o socialización) y para que ello ocurra, es necesario el lenguaje y la comunicación que logra alcanzar niveles de interpretación y comprensión, a través de la codificación de palabras, gestos y símbolos que en su intercambio surge un nivel de comprensión compartida y le da nuevo significado a la situación estudiada.

- Teoría fundada: según Barney Glaser y Strauss (1983) ayuda a contribuir a cerrar la brecha entre la teoría y la investigación empírica. Es una metodología que se desarrolla a partir de datos que son sistemáticamente capturados a través de la forma de pensar, de las formas de conceptualizar los hechos y las teorías que involucran la situación social.

Esta teoría tiene la posibilidad de dar cuenta de las realidades humanas singulares, porque se alimenta 
La investigación cualitativa en educación y la relación con el campo pedagógico Alberto Sarmiento Castro

principalmente de la recolección de los datos dentro de la investigación misma, de manera dinámica y abierta; es inductiva y va buscando caminos que permita construir la teoría que le de significado no sólo a los datos sino al análisis mismo como un propósito explicito de hacer posible la verificación de las hipótesis que surgen (teoría fundada).

Este método se basa en cuatro estrategias principalmente: 1. Un interrogatorio sistemático a través de preguntas generativas que buscan principalmente relacionar conceptos; 2. El muestreo teórico; 3. Los procedimientos de categorización (codificación sistemática); 4. El seguimiento de los principios dirigidos a conseguir un desarrollo conceptual sólido; por lo tanto, no basta sus afirmaciones en la descripción sólo de los hechos.

A través del artículo se establece una disertación y argumentación para el fortalecimiento continuo de los documentos de cada uno de los autores que han construido o han ido construyendo la teoría de la investigación cualitativa en el campo educativo y desde el contexto de la acción pedagógica se pueda establecer y determinar la importancia que tiene la investigación cualitativa en la educación, en especial los fenómenos que encierra los campos de la pedagogía y los aportes que ha venido construyendo a lo largo del desarrollo del espacio investigativo de los fenómenos humanos; pero principalmente, busca que las personas, los profesores y intelectuales de la educación en todos y en cada uno de los niveles, asuman su propio rol de investigadores, tracen para utilizar uno $u$ otro paradigma de la investigación social, que encausen los interrogantes del problema educativo frente a la enseñanza, el aprendizaje y las acciones desarrolladas por maestros y alumnos que buscan permanente signos y niveles de interpretación, explicación y comprensión del acto pedagógico en el campo educativo, social, cultural y político que vive el país.

\section{REFERENCIAS BIBLIOGRÁFICAS}

AGUIRRE BAZTÁN, Ángel. Etnografía. México: Alfaomega y Marcombo, 1995.

BRIONES, Guillermo. La investigación en el aula y la escuela. $3 \mathrm{Ed}$. Santafé de Bogotá: Convenio Andrés Bello, 1996.

CAJIAO RESTREPO, Francisco. Selene. Segunda expedición de pleyade. Fundación FES. MEN. Santafé de Bogotá. 1998.

CARRASCO, José Fernando y CALDERO HERNANDÉZ, José Fernando. Aprendo a Investigar en Educación. Madrid. Ediciones Rialp s.a. 2000.

CERDA GUTIÉRREZ, Hugo. La investigación total. 2 Ed. Santafé de Bogotá: Magisterio, 2001.

CERDA GUTIÉRREZ, Hugo. Los elementos de la investigación. Santafé de Bogotá. Editorial el Buho. 1.995

CLAUDE LEVI-Straus. la mirada distante. Barcelona Argos Vergara, 1889.

DILTHEY, Wilhelm, Introduccción a las ciencias del espíritu. Editorial Alianza, Madrid, 1980.

GEERTZ. la interpretación de las culturas. México, Gedisa, 1973.

GODOMER, Verdad y Métodos, Salamanca, 1977

GLASSER, Barney y Straus, Anselm L. Theory Strategies for Qualitative Reseorch, Chicago: Aldine de Gruyter

LAMUS CAÑAVATE, Doris. Seminario investigación social cualitativa. Bucaramanga: U.I.S., Facultad de Ciencias Humanas, Maestría en Pedagogía, 2003. 
La investigación cualitativa en educación y la relación con el campo pedagógico

Alberto Sarmiento Castro

SÁNCHEZ GAMBOA, Silvio. Fundamentos para la investigación educativa. Santafé de Bogotá: Magisterio, 1999.

SANDOVAL CASILIMAS, Carlos Arturo. Seminario investigación educativa. Bucaramanga: U.I.S., Facultad de Ciencias Humanas, Maestría en Pedagogía, 2003.

SARMIENTO CASTRO, Alberto. Criterios de Investigación. UNIVERSIDAD FRANCISCO DE PAULA SANTANDER. Facultad de Educación, Artes y Humanidades. Departamento de Humanidades. San José de Cúcuta. 2.001.

TAYLOR. S.J y BOGDAN. R. introducción a los métodos cualitativos de investigación. Barcelona. Paidós. 1994. 